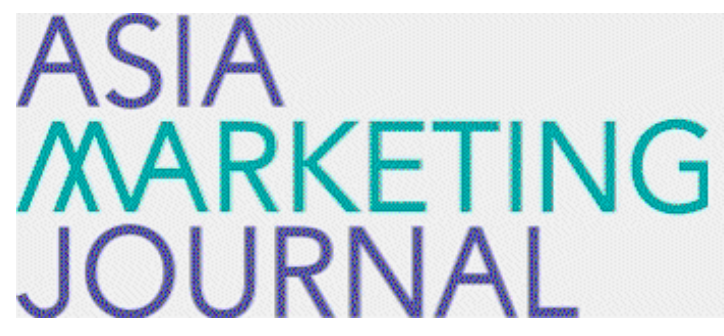

ASIA MARKETING JOURNAL

Volume 22 | Issue 1

Article 3

4-30-2020

\title{
Effect of Social Norm on Consumer Demand: Multiple Constraint Approach
}

Sungjee Choi

Inwoo Nam

Jaehwan Kim

Follow this and additional works at: https://amj.kma.re.kr/journal

Part of the Marketing Commons

\section{Recommended Citation}

Choi, Sungjee; Nam, Inwoo; and Kim, Jaehwan (2020) "Effect of Social Norm on Consumer Demand: Multiple Constraint Approach," Asia Marketing Journal: Vol. 22 : Iss. 1 , Article 3.

Available at: https://doi.org/10.15830/amj.2020.22.1.41

This Article is brought to you for free and open access by Asia Marketing Journal. It has been accepted for inclusion in Asia Marketing Journal by an authorized editor of Asia Marketing Journal. 


\title{
Effect of Social Norm on Consumer Demand: Multiple Constraint Approach*
}

\author{
Sungjee Choi** \\ Inwoo Nam*** \\ Jaehwan Kim****
}

The goal of the study is to understand the role of social norm in purchase decisions where demand is revealed in the form of multiple-discreteness. Consumers are socially engaged in various activities through the expectation from others in their community. Actions or decisions are likely to reflect this influence. This implicit or explicit social norm is revealed as the rules, regulations, and standards that are understood, shared, endorsed, and expected by group members. When consumers' decisions are in distance from the norm, they come to face discomfort such as shame, guilt, embarrassment, and anxiety. These pressure act as a constraint as opposed to utility in their decision making.

In this study, the effect of social norms on consumer demand is captured via multiple constraint model where constraints are not only from budget equation but also from psychological burden induced by the deviation from the norm. The posterior distributions of model parameters were estimated via conjoint study allowing for heterogeneity via hierarchical Bayesian framework. Individual characteristics such as age, gender and work experience are also used as covariates for capturing the observed heterogeneity. The empirical results show the role of social norm as constraint in consumers' utility maximization. The proposed model accounting for social constraint outperforms the standard budget constraint-only model in terms of model fit. It is found that people with longer job experience tend to be more robust and resistant to the deviation from the norm. Incorporating social norm into the utility model allows for another means to disentangle the reason for no-purchase as 'not preferred' and 'not able to buy'.

Keywords: Social norms, Constraint, Choice model, Multiple discreteness, Bayesian estimation

\footnotetext{
* This research was supported by Korea University Research Grant.

** Tippie College of Business, University of Iowa (sungjee-choi@uiowa.edu)

*** School of Business Administration, Chung-Ang University (inam@cau.ac.kr)

**** Korea University Business School (jbayes@korea.ac.kr), Corresponding Author
} 


\section{Introduction}

Social norm is a set of specific behavioral patterns that are shared and accepted within a group in a particular situation. Human beings are essentially social beings and, in most cases, they are sensitive to social influence. As the behavior of others often acts as a guide to their own behavior (Gockeritz et al. 2010, Brewer and Hewstone 2004), they are often observed to be subject to social norms in their purchase decision as well. For example, an individual who originally prefers bright pink suits and yellow pants may hesitate to purchase them when she is aware of possible negative reactions from peers or parents. So the pressure can alter her final choice to one not predicted by her original preference.

In this study, we measure the effect of social pressure driven by the deviation from norms on demand when consumers are constrained utility maximizers. Consumers trade off the comfort in their mind and the level of maximum attainable utility, implying that social norm enters decision as constraint rather than utility.

What consumers must give up for acquiring goods they prefer is defined as constraint which is mostly represented by budget constraint in the literature. There are quite a few examples that consumer demand is also influenced by other constraints and restrictions, which are nothing to do with monetary budget. Previous studies documented the existence of these non-monetary constraints including mental constraints (Kim et al. 2017), storage constraints (Satomura et al. 2011) and so on.

The observed demand in the market place are attributed to both consumers' preference and constraint. Therefore, in economic theory, zero-purchase is due to 'not preferred enough', 'not able to tolerate the cost', or both. The burden induced by the deviation from social norm is naturally akin to cost side. Therefore, the proper approach to quantify the effect of social norm on demand is to allow this pressure to enter as a cost component in consumers' utility maximizing decision.

Suppose that sales of a red car are very low in a given market area, specific customer segment. This may be due to the low preference for red car, i.e., the buyers in this segment like red less than black, or higher pressure associated with buying red car, i.e., peer effect on color choice. When low or zero demand is observed in the market, therefore, it is critical to empirically measure whether the low sales is from lower preference or higher social burden in more structured manner. For this purpose, we use direct utility model approach with multiple constraint specification. Direct utility framework has been widely applied in literature to flexibly capture the demand in both corner and interior solutions(e.g., Kim et al. 2002; Bhat 2005, 2008; Lee et al. 2013; Luo et al. 2013; Lin et al. 2013). The model specification allows for 
separating the cost effect from the utility effect in consumer demand. We incorporate social norm into the model specification as additional constraint(e.g. Satomura et al 2011, Kim et al 2017). We let the marginal utility be also function of the product attributes so that one can still capture the utility change due to product attributes. We also empirically investigate how individual characteristics (age, gender and work experience) are related to preference for product offerings when consumers do consider social norm in their constrained utility maximization.

The remainder of the paper is organized as follows: In section 2, conceptual background for the study including the definition of social norms is given. We show model specification, likelihood, and heterogeneity in section 3. In section 4, we discuss the data and estimation results. Then we explore two managerial implications from our study in section 5 and we offer concluding remarks with opportunities for future study in section 5 .

\section{Conceptual Background}

\subsection{Literature}

Not to mention Ajzen and Fishbein(1973), the role of social norm and its effect on our behavior has been well documented in previous studies. The applications are various ranging from college health care marketing to individuals volunteering decisions in literature. Haines and Spear (1996) demonstrated that 'social norms marketing' is of help to lower alcohol consumption on campus. Similarly, social norm marketing is an effective tool to change children's perceptions of tobacco usage (Sheikh et al. 2017). Harries et al. (2013) reported that people reduce electricity consumption more when they receive information about others' consumption than when they got their own consumption record.

Not only consumption decisions, but also nonpurchase/acquisition decisions were associated with the social norm, including moral judgement, donation, boycott, etc. Fisher and Ackerman (1998) found that social norms appeal to individuals' volunteering decision. Sen et al. (2001) showed peoples' participation rate on boycott depends on their personality toward the society.

These studies in literature give an immediate intuition to marketing area that consumers' purchase decisions are largely influenced by other people. Many studies in marketing literature also dealt with the social interactions, covering 'word of mouth (WOM)', 'peer influence', 'contagious group', etc.

Despite the advances made to date, the results reported in literature are based on mixed usage in terms of the aspect of the social norm itself as a construct and the level of behavior set to be affected by norm. For example, norms can be formal and informal. Or some are inductive 
and some are descriptive. It may merely set an expectation that members change the attitude, or require a specific level of adjustment for revealed behavior. Some norms are originally formed with specific intention such as code of conduct imposed by an organization. In contrast, other types of norms are naturally developed in the market place, e.g., a salient consumption trend that gives rise to dominating repattern as more people comes to market via imitation.

Therefore, we first clarify the scope of the social norm used in this study in order to investigate its role in demand formation. In next sections, we elaborate more about the scope of social norm used in the study and basic quantitative specification for how the social norm enters in their decision making. The premise is that social norm influences individuals' demand through the psychological burden associated with buying goods.

\subsection{Conceptual Framework of Social Norm}

Social norm is a set of specific behavioral patterns that are shared and accepted within a social group in a particular situation. Often it is also defined as attitudes and behaviors that are expected and required by society in specific situations (Baron et al. 1992, Leon et al. 1950).

The framework for conceptualizing the social norm adopted in this study is based on two criteria - one is form of the requirement that originates from the norm, and the other is the level to which individuals are to conform. The framework is described in figure 1, which also comes from refining Paluck and Ball (2010).

The first criterion, form, is about whether the social norm imposes explicit/formal requirement or it is given to individuals as implicit/informal expectation that members had better conform to. The difference between the two forms is the type of punishment one would receive as

〈Figure 1〉 Framework of social norms

Form presented to individuals

\begin{tabular}{|c|c|c|}
\hline \multirow[b]{2}{*}{ Level of adjustment } & \\
\hline & $\begin{array}{c}\text { Expectation } \\
\text { (implicit/informal) }\end{array}$ & $\begin{array}{c}\text { Requirement } \\
\text { (explicit/formal) }\end{array}$ \\
\hline $\begin{array}{l}\text { Behavior } \\
\text { (revealed) }\end{array}$ & $A^{*}$ & B \\
\hline $\begin{array}{c}\text { Attitude } \\
\text { (not revealed) }\end{array}$ & $\mathrm{C}$ & $\mathrm{D}$ \\
\hline
\end{tabular}


a consequence of the violation. Norms formally required of the members are often associated with practical punishment such as a demotion at work, getting bad grade in a class, or legal punishment. On the other hand, failure to meet the expected social norm brings about implicit punishment such as sense of guiltiness and other people's negative gaze.

The second criterion is as to whether the adjustment to be made is via consumers' behavior or at the attitude level. The difference between behavior and attitude is that one is revealed and the other is not. Attitude is related to mind and thinking, so it is not revealed and unobservable in the market. Examples are having generosity to the people who are in need, having respect to retired veteran, etc. In contrast, behavior is directly observable in most cases. Refraining self from buying a car with specific color, not speaking loudly in public place, and so on are the examples for the level at which social norm interferes the behavior.

As the results, there are four types of social norms - behaviors expected(A) or required(B), and attitudes one think the society to expect to possess (C) or to require to hold(D). Among the four combinations, we focus on the first case in which consumers consider whether their behaviors are consistent with the social expectations (i.e., A). It is because the focus of the study is behavior rather than attitude, and we use observed data on consumer's choice/ quantity decision in empirical analysis. Another reason is that we analyze individuals' decision when they recognize an implicit norm $(A)$, rather than a stated formal rule(B and D). In 'A', discrepancy from the norm gives a discomfort although it does not necessarily incur the formal punishment. In this study, the choice of business wear at work place is used as buyers' decision variables, which will be described in more detail in the empirical analysis section.

What ultimately matters in consumers' final decision would not be social norm itself. Instead, it is their pressure due to the gap between intended decision and the one that is socially desirable. For example, I plan to purchase a red \& luxurious car, but it's way different from my significant others' thoughts. Although I don't have any formal obligation to follow their expectation, I may avoid the red color and adjust to downgrading of the car in the end.

This distance between the desirable point imposed by social norm and the actual point obtained from actual product choice enters as cost or expense that individuals have to take as they maximize the utility from purchase. Kreps (1997) mentioned that social norms constrain people's behavior, and it can be studied in the utility maximization framework. Paluck and Ball (2010) argued that social norms act as a powerful constraint on individual attitudes and behaviors. In next section, therefore, we model the role of social norm as an additional source of constraint, separating from the existing budget constraint. 


\section{Model}

\subsection{Basic Structure}

Following the discussion in 2.1 and 2.2, the model structure is given as below. The model can deal with an arbitrary number of products and type of demand, i.e., discrete or continuous, and it is based on the utility maximization with double constraints employed in literature (e.g., Satomura et al. 2011) :

$$
\begin{aligned}
& \max \mathrm{U}\left(x_{k t}\right) \\
& \text { subject to } \sum_{n=1}^{N} p_{k n t} x_{k n t}+x_{k q t}=M_{k} \\
& \sum_{n=1}^{N} w_{k n} x_{k n t}+x_{k c t}=S_{k}
\end{aligned}
$$

where

$$
\begin{aligned}
& \mathrm{k}: \text { individual } \mathrm{k}(\mathrm{k}=1, \ldots, \mathrm{K}) \\
& \mathrm{n}: \operatorname{good} \mathrm{n}(n=1, \ldots, \mathrm{N}) \\
& \mathrm{t}: \text { time at } t(t=1, \ldots, \mathrm{T})
\end{aligned}
$$

An individual consumer $k$ is assumed to make her decision on what to buy and how much to buy over $\mathrm{N}$ items $\left(x_{n}, n=1, \ldots, N\right)$ in the focal category, and how much to spend on outside goods $\left(x_{q}, x_{c}\right)$. An arbitrary utility function $\mathrm{U}(\mathrm{x})$ is maximized subject to budget constraint in (1) and social norms constraint in (2):

In the budget constraint, there is $p_{n}$, the price of item $\mathrm{n}$, and $x_{n}$, the quantity demanded.
The total expenditure over $\mathrm{N}$ items $\left(\sum_{n} p_{k n} x_{k n}\right)$ cannot exceed $M_{k}$, the consumer $k$ 's maximum budget. The second constraint in (2) is about the individual's capacity to tolerate social pressure. Here, $w_{n}$ is marginal social burden or concern that one has to take for each additional quantity of good $n$. Both $p_{n}$ and $w_{n}$ are observed from data. If an individual feels that the consuming good $n$ is uncomfortable against her social norm, $w_{n}$ is large to her. Similar to budget constraint, $\sum_{n} w_{k n} x_{k n}$ is a total inconvenience from consuming goods in the category, and it does not exceed $S_{k}$, the individual k's psychological capability to tolerate the discomfort. One assumption of the model is that consumers do not exhaust their budgetary allotment nor their psychological capacity. So $x_{q}$ and $x_{c}$ are outside goods for budget and social norm constraint, respectively, and both are assumed strictly positive demand.

\subsection{Utility function}

For application, we use the direct utility specification for $\mathrm{U}(\mathrm{x})$ in 3.1 This framework can handle the simultaneous demand for varieties allowing for both corner and interior solution.

$$
\begin{gathered}
\mathrm{U}\left(x_{k t}\right)=\sum_{n=1}^{N} \psi_{k n t} \log \left(x_{k n t}+1\right) \\
+\psi_{k q} \log \left(x_{k q t}\right)+\psi_{k c} \log \left(x_{k c t}\right)
\end{gathered}
$$


The utility is expressed log-linearly to capture the diminishing returns to marginal utility, which allows for interior solutions. Parameter $\psi_{k n t}$ is the baseline utility of good $n$ for individual $k$ at time $t$. Two outside goods are also added, $x_{k q t}$ and $x_{k c t}$ from constraints in (1) and (2), respectively. Thus the marginal utility parameters for both outside goods are fixed to 1 for identification purpose, i.e., $\psi_{k q}=$ $1, \psi_{k c}=1$. The translation by " +1 " in $\left(x_{k n t}+1\right)$ assures the existence of corner solution so that the marginal utility at $x_{n}=0$ is finite.

We relate baseline preference parameter for good $n$ to the vector of its product attribute $\left(a_{n}\right)$. The randomness $\left(\varepsilon_{k n t}\right)$ enters the marginal utility via $\psi$ parameter, and is assumed to Type I extreme value distributed:

$$
\begin{aligned}
\psi_{k n t}= & \exp \left(\psi_{k n}+\varepsilon_{k n t}\right), \\
& \varepsilon_{k n t} \sim \text { i.i.d } \operatorname{EV}(0,1) \\
\psi_{k n}= & \psi_{k 0, n}+\sum_{l=1}^{L} \beta_{k l} a_{n l}
\end{aligned}
$$

where $a_{n l}$ is the $l^{t h}$ attribute of good $\mathrm{n}$ and $\beta_{k l}$ is the corresponding coefficient.

\subsection{Likelihood}

The likelihood for the observed demand from the constrained utility maximization is derived by forming a Lagrangian equation for the optimality condition and incorporating the statistical distribution over the random terms ( $\varepsilon$ 's). First, we form an auxiliary equation that combines the utility function in (3) with Lagrangian multipliers $\left(\lambda_{1}, \lambda_{2}\right)$ for each of the constraints:

$$
\begin{aligned}
L= & U\left(x_{k t}\right)+\lambda_{1}\left(M_{k}-\sum_{n=1}^{N} p_{k n t} x_{k n t}-x_{k q t}\right) \\
& +\lambda_{2}\left(S_{k}-\sum_{n=1}^{N} w_{k n} x_{k n t}-x_{k c t}\right) .
\end{aligned}
$$

We assume the outside goods are strictly positive values, so the two constraints are always binding, indicating that $\lambda_{1}$ and $\lambda_{2}$ are positive. By rewriting the expressions for complementary slackness, we have following stochastic Kuhn-Tucker conditions associated with the first-order conditions of the observed demand:

$$
\begin{aligned}
\frac{\partial L}{\partial x_{k n t}} & =\frac{\psi_{k n t}}{\psi_{k n t}+1}-\frac{\psi_{k q} \cdot p_{k n t}}{x_{k q t}}-\frac{\psi_{k c} \cdot w_{k n}}{x_{k c t}} \\
& \left.=0, \text { and } x_{k n t}\right\rangle 0 \\
\frac{\partial L}{\partial x_{k n t}}= & \frac{\psi_{k n t}}{\psi_{k n t}+1}-\frac{\psi_{k q} \cdot p_{k n t}}{x_{k q t}}-\frac{\psi_{k c} \cdot w_{k n}}{x_{k c t}} \\
& \left\langle 0 \text {, and } x_{k n t}=0\right.
\end{aligned}
$$

Taking logarithms of (6) and (7), we have:

$$
\begin{aligned}
& \varepsilon_{k n t}=g_{k n t} \text { if } \quad x_{k n t}>0 \\
& \varepsilon_{k n t}\left\langle g_{k n t} \text { if } \quad x_{k n t}=0\right.
\end{aligned}
$$

where

$$
\begin{aligned}
& g_{k n t}=-\psi_{k n}+\log \left(x_{k n t}+1\right)+ \\
& \log \left(\frac{\psi_{k q} \cdot p_{k n t}}{M_{k}-\sum_{n=1}^{N} p_{k n t} x_{k n t}}+\frac{\psi_{k c} \cdot w_{k n}}{S_{k}-\sum_{n=1}^{N} w_{k n} x_{k n t}}\right)
\end{aligned}
$$

The distributional property of $\varepsilon_{k n t}$ in $\mathrm{KT}$ 
equalities and inequalities in (8) and (9) gives rise to the statistical likelihood for any type of observed demand at a time $t$ in closed form. Suppose that $\mathrm{R}$ goods are purchased out of total $\mathrm{N}$ goods and the data points are rearranged in the order that non-zero $\operatorname{demand}(\mathrm{x}>0)$ comes first and no-purchase $(\mathrm{x}=0)$ comes later. Then, the likelihood for the observed demand $\left(x_{k t}\right)$ is:

$$
\begin{aligned}
& P\left(x_{k t}\right) \\
= & P\left(x_{k i t}>0 \text { and } x_{k j t}=0 ;\right. \\
& i=1, . ., R \text { and } j=R+1, . ., N) \\
= & \left|J_{k R t}\right| \int_{-\infty}^{g_{k N t}} \ldots \int_{-\infty}^{g_{k, R+1, t}} \\
& \quad f\left(g_{k 1 t}, \ldots, g_{k R t}, \epsilon_{k R+1 t}, \ldots, \epsilon_{k N t}\right) d_{\epsilon_{k R+1 t}}, \ldots, d_{\epsilon_{k N t}} \\
= & \left|J_{k R t}\right|\left\{\prod_{i=1}^{R} \exp \left(-g_{k i t}\right)\right\} \\
& \cdot \exp \left\{-\sum_{j=1}^{N} \exp \left(-g_{k j t}\right)\right\}
\end{aligned}
$$

In this likelihood, the KT equalities for nonzero demand in (8) requires the density evaluation of $f(\cdot)$ and $\mathrm{KT}$ inequalities for zero demand in (9) are expressed as probability mass. The term $\left|J_{k R t}\right|$ is the RxR Jacobian for interior solutions mapping random-utility $\operatorname{error}(\varepsilon)$ to the observed $\operatorname{data}(x)$ :

$$
\begin{aligned}
J_{k R t[i, j]}= & \frac{\delta_{i j}}{x_{k j t}+1} \\
& +\frac{p_{k i t} \cdot p_{k j t} / x_{k q t}^{2}+w_{k j} \cdot w_{k i} / x_{k c t}^{2}}{p_{k i t} / x_{k q t}+w_{k i} / x_{k c t}}
\end{aligned}
$$

where $\delta_{i j}=1$ if $i=j$, and $\delta_{i j}=0$ otherwise.

\subsection{Heterogeneity}

The parameters in the likelihood in (11) are individual-specific, and they are all estimated with heterogeneity across people in the data. The vector of parameters for consumer $\mathrm{k}, \boldsymbol{\theta}_{k}$ in the estimation is:

$$
\boldsymbol{\theta}_{k}=\left(\psi_{k 0}^{\prime}, \boldsymbol{\beta}_{k}^{\prime}, M_{k}^{*}, S_{k}^{*}\right)^{\prime}
$$

where $M_{k}^{*}=\ln \left(M_{k}\right)$ and $S_{k}^{*}=\ln \left(S_{k}\right)$ to assure the positivity of the resource parameters, i.e., $0<M_{k}, S_{k}$. We relate these model parameters to each individual characteristic for capturing the observed heterogeneity, and assume the unobserved heterogeneity component to be normally distributed as given in (13):

$$
\theta_{k}=\Delta^{\prime} z_{k}+\zeta_{k}, \zeta_{k} \sim \mathrm{N}\left(0, V_{\theta}\right)
$$

where $z_{k}$ is the vector of consumer k's individual-level covariates, $\Delta$ is the coefficient matrix measuring the relationship between $\mathrm{Z}$ and $\theta$, and $\boldsymbol{V}_{\theta}$ is the covariance matrix reflecting the unobserved heterogeneity.

\section{Empirical Analysis}

\subsection{Data Description}

The data used in the analysis were about 
choice of shirts that people would wear at work, and collected by choice-based conjoint survey in South Korea. Wearing shirts at work has been mostly expected for a long time. In the study, participants were told a scenario that:

their workplaces have a bylaw to wear a suit, there is no mandatory guideline for color or pattern of the shirts; most of their colleagues at work usually wear white shirts with no pattern.

Then participants had ten choice tasks. At each task, they were instructed to show what and how many to purchase among eight varieties of shirts without any restriction on their choice whether it is to be corner or interior solution. Product attributes and varieties used in data collection were given in table 1 (a) and (b).
Among the profiles used in the study, the product with $\left(a_{1}=0, a_{2}=0\right)$, i.e., white shirts $\left(a_{1}=0\right)$ with no $\operatorname{pattern}\left(a_{2}=0\right)$ is the one conforming to the norm descried in the scenario. The prices $\left(p_{k n t}\right)$ and the order of the shirts were changed across choice tasks. At the end of the session, participants were asked about the inconvenience they may have with each $\operatorname{shirt}\left(w_{k n}\right)$ if they wore it. Three variables $\left(z_{1}\right.$, $\left.Z_{2}, Z_{3}\right)$ were recorded for individual characteristics including age $\left(z_{1}\right)$, gender $\left(z_{2}=0\right.$ if female, 1 otherwise), and work experience $\left(z_{3}=1\right.$ if experience, 0 otherwise) for each of the total 104 individuals. The average age of the participants was 25.6. Total 68(36) respondents were female(male) and those who replied they had work experience was 56 .

〈Table 1〉 Product offerings and attributes

(a) Attributes coding

\begin{tabular}{cccc}
\hline Attribute & Coding & $\mathrm{a}_{1}$ & $\mathrm{a}_{2}$ \\
\hline \multirow{2}{*}{ Color } & White & 0 & \\
& Non-white & 1 & \\
\hline \multirow{2}{*}{ Pattern } & No & & 0 \\
& Yes & & 1 \\
\hline
\end{tabular}

(b) Varieties and attribute combinations

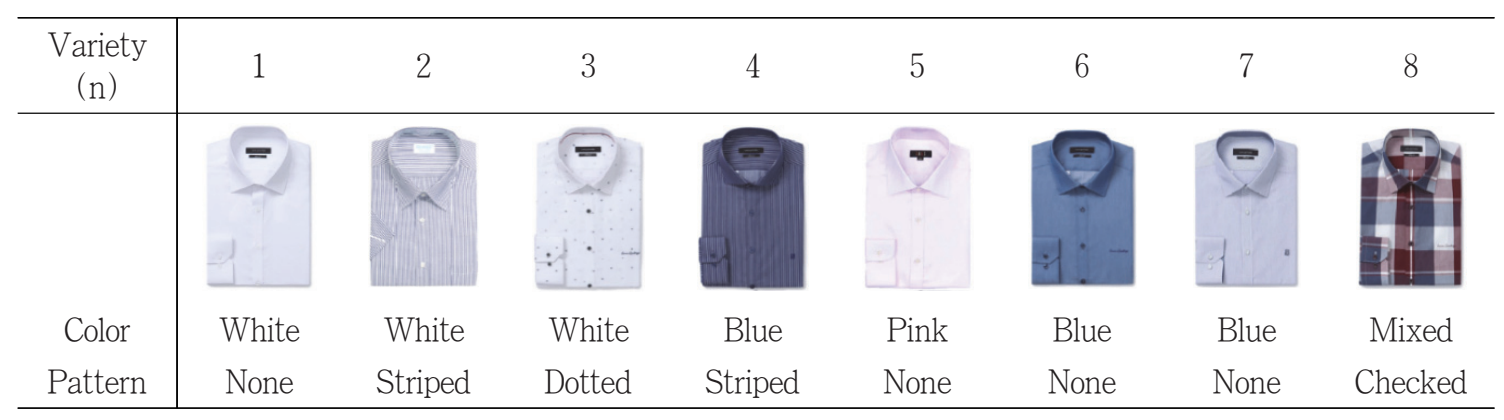


〈Table 2〉 Purchase incidence, quantity, and type of purchase

\begin{tabular}{cccccc}
\hline $\begin{array}{c}\text { Variety } \\
(\mathrm{n})\end{array}$ & $\begin{array}{c}\text { Purchase } \\
\text { incidence }\end{array}$ & $\begin{array}{c}\text { Purchase } \\
\text { quantity }\end{array}$ & $\begin{array}{c}\text { Single item purchase } \\
\text { (Corner Solution) }\end{array}$ & $\begin{array}{c}\text { Multiple items purchase } \\
\text { (Interior Solution) }\end{array}$ & $\begin{array}{c}\text { Interior Solution } \\
\text { Proportion }\end{array}$ \\
\hline 1 & 820 & 2092 & 96 & 724 & 0.88 \\
2 & 301 & 376 & 6 & 295 & 0.98 \\
3 & 176 & 255 & 0 & 176 & 1.00 \\
4 & 253 & 315 & 4 & 249 & 0.98 \\
5 & 240 & 331 & 1 & 239 & 0.99 \\
6 & 398 & 540 & 14 & 384 & 0.96 \\
7 & 510 & 745 & 3 & 507 & 0.99 \\
8 & 81 & 105 & 2 & 79 & 0.98 \\
\hline Total & 2779 & 4759 & 126 & 2653 & 0.95 \\
\hline
\end{tabular}

Table 2 provides the overall purchase pattern observed in our data. Participants purchased variety \#1 the most (820 times) whereas they chose \#8 the least (81 times). As the mean proportion of the interior solution is $95 \%$, respondents chose multiple shirts at a time for their use at work. We will explore whether the observed differences among varieties in table 2 is from preference difference or something else such as psychological burden due to not conforming to the implicit social norm in our data. So we first check the model fit and discuss parameter estimates in next section, and later we quantify the effect of social burden in comparison with the effect of price cut on consumer welfare.

\subsection{Estimation Results}

The joint posterior distribution of the model parameters was estimated via Markov chain
Monte Carlo (MCMC) methods in hierarchical Bayesian framework. The recursive sampling scheme is:

$$
\theta_{k} \mid \triangle, V_{\theta}, z_{k}, x_{k} \text { for } \mathrm{k}=1, \cdots, \mathrm{K}
$$

$$
\begin{gathered}
\Delta \mid\left\{\boldsymbol{\theta}_{k}, \mathrm{k}=1, \cdots, \mathrm{K}\right\}, \boldsymbol{V}_{\theta}, z_{k} \\
\boldsymbol{V}_{\theta} \mid\left\{\boldsymbol{\theta}_{k}, \mathrm{k}=1, \cdots, \mathrm{K}\right\}, \Delta, z_{k}
\end{gathered}
$$

Metropolis-Hastings random work chain was used for obtaining posterior distribution of individual parameters, $\theta_{k}$ for all $\mathrm{k}$. Standard Gibbs sampler was used for estimating $\Delta$ (normal posterior) and $V_{k}$ (Inverted Wishart posterior) with diffuse priors. Every $10^{\text {th }}$ draw out of total 40,000 iterations were retained to control autocorrelations, and last 15,000 iterations after burn-in were used for producing posterior summary. The detailed estimation outcome is available from authors upon request. 


\subsubsection{Model Fit}

We first check whether consumers' responses reflect the effect of social norm as another source of burden in addition to monetary constraint in the current data. For this purpose, we fit an alternative model as a benchmark consists of the same utility specification in (3) but without social norm constraint in (2), i.e.,

$$
\begin{gathered}
\max \mathrm{U}\left(x_{k t}\right) \sum_{n=1}^{N} \psi_{k n t} \log \left(x_{k n t}+1\right) \\
+\psi_{k q} \log \left(x_{k q t}\right)
\end{gathered}
$$

subject to $\sum_{n=1}^{N} p_{k n t} x_{k n t}+x_{k q t}=M_{k}$

In table 3 , log marginal densities for the benchmark model and proposed models are reported. The model fit comparison shows that the proposed model outperforms the budget constraint-only model, implying that the observed demand reflects the role of social norm as additional constraint. In next section, therefore, we discuss the parameters estimates focusing on the proposed model first, and explore the possible bias when one fails to ignore the social constraint.

〈Table 3〉 Model fit comparison

\begin{tabular}{c|c}
\hline Model & Log-marginal density \\
\hline Benchmark & -6343.69 \\
Proposed & -5825.04 \\
\hline
\end{tabular}

\subsubsection{Parameter Estimates}

Posterior mean and standard deviation for the

$\langle$ Table 4$\rangle$ Posterior mean of coefficient $\left(\Delta^{\prime}\right)$ and unobserved heterogeneity $\left(V_{\theta}\right)$

\begin{tabular}{c|cccc|c}
\hline & Intercept & Age & Gender & Work experience & Heterogeneity $\left(V_{\theta[i, i]}\right)$ \\
\hline$\psi_{0,1}$ & 0.081 & -0.059 & -0.022 & 0.371 & 2.412 \\
$\psi_{0,2}$ & -0.211 & -0.029 & -0.089 & 0.164 & 1.438 \\
$\psi_{0,3}$ & -0.504 & -0.020 & 0.267 & 0.333 & 1.390 \\
$\psi_{0,4}$ & -0.011 & 0.024 & -0.215 & -0.099 & 1.107 \\
$\psi_{0,5}$ & -0.426 & -0.015 & -0.400 & 0.378 & 1.381 \\
$\psi_{0,6}$ & -0.170 & -0.007 & 0.507 & -0.013 & 0.039 \\
$\psi_{0,7}$ & 0.025 & -0.024 & -0.043 & -0.031 & 1.393 \\
$\psi_{0,8}$ & -0.341 & -0.002 & 0.393 & -0.040 & 1.084 \\
$\beta_{1}$ & -0.867 & -0.014 & 0.112 & 0.272 & 1.504 \\
$\beta_{2}$ & -1.060 & -0.011 & 0.065 & 0.155 & 1.307 \\
$M^{*}$ & 5.790 & 0.004 & 0.046 & 0.175 & 0.425 \\
$S^{*}$ & 1.293 & -0.032 & 0.503 & 0.756 & 2.113 \\
\hline
\end{tabular}

Note. Estimates where 95\% credible region does not include zero are in bold. 
parameters and hyper parameters are reported in table 4 . First, the utility effects of non-white color $\left(a_{1}=1\right)$ and being patterned $\left(a_{2}=1\right)$ are negative as $\beta_{1}<0$ and $\beta_{2}<0$. In the data, respondents' preference for shirt is lowered if there's any pattern in the shirt or the color is non-white. Relative to the first $\operatorname{shirt}\left(\psi_{0,1}\right)$, the intercept in baseline preference for rest of the shirts are low. Secondly, the log of budget limit $\left(\mathrm{M}^{*}\right)$ and psychological capacity $\left(\mathrm{S}^{*}\right)$ parameters are estimated to be on average 5.970 and 1.293, respectively.

From the diagonal elements of $V_{\theta[i, i]}$, there's substantial amount of heterogeneity in parameters across consumers. These individual-level parameters are not significantly related to consumers' demographic variables such as age $\left(z_{1}\right)$ and gender $\left(z_{2}\right)$. It is notable, however, that the relationship between $\mathrm{S}^{*}$ and work-experience $\left(z_{3}\right)$ is significant (0.756). Those who have work experience tend to have larger psychological capacity to handle the social pressure. A more detailed discussion for parameter estimates is given in the next section via the comparison across the budget constraint model.

\subsubsection{Bias in utility parameters due to ignoring the social constraint}

What would be the bias when social constraint is not considered in the model? How would marketers' understanding on consumer preference would be biased when they ignore the role of social norm-constraint despite the presence of multiple constraint in the demand? In order to address this question, we compare the parameter estimates between the proposed model and the single constraint model in (14).

〈Table 5〉 Comparison in utility parameters

\begin{tabular}{cccc}
\hline Parameter & $\begin{array}{c}\text { Proposed } \\
\text { Model }\end{array}$ & $\begin{array}{c}\text { Benchmark } \\
\text { Model }\end{array}$ \\
\hline & $\overline{\psi_{1}}$ & 0.08 & -0.10 \\
& $\overline{\psi_{2}}$ & -1.28 & -2.04 \\
Baseline & $\overline{\psi_{3}}$ & -1.57 & -2.44 \\
Preference & $\overline{\psi_{4}}$ & -1.95 & -2.96 \\
& $\overline{\psi_{5}}$ & -1.30 & -2.15 \\
& $\overline{\psi_{6}}$ & -1.05 & -1.79 \\
& $\overline{\psi_{7}}$ & -0.85 & -1.36 \\
& $\overline{\psi_{8}}$ & -2.28 & -3.52 \\
\hline Attribute & $\overline{\beta_{1}}$ & -0.87 & -1.42 \\
effect & $\overline{\beta_{2}}$ & -1.07 & -1.65 \\
\hline
\end{tabular}

In table 5, we report the mean of baseline preference for each of the shirt varieties $\left(\overline{\psi_{1}}\right.$, $\ldots, \overline{\psi_{8}}$ ) and the mean of preference impact of $\operatorname{attributes}\left(\overline{\beta_{1}}, \overline{\beta_{2}}\right)$ after integrating across individuals and over the data for each model. First, the baseline parameters from the bench mark model are systematically lower than those of the proposed model, indicating there's downward bias in the preference when ignoring the role of additional constraint. In the benchmark model with budget constraint only, the effect of social constraint is absorbed by the preference, 


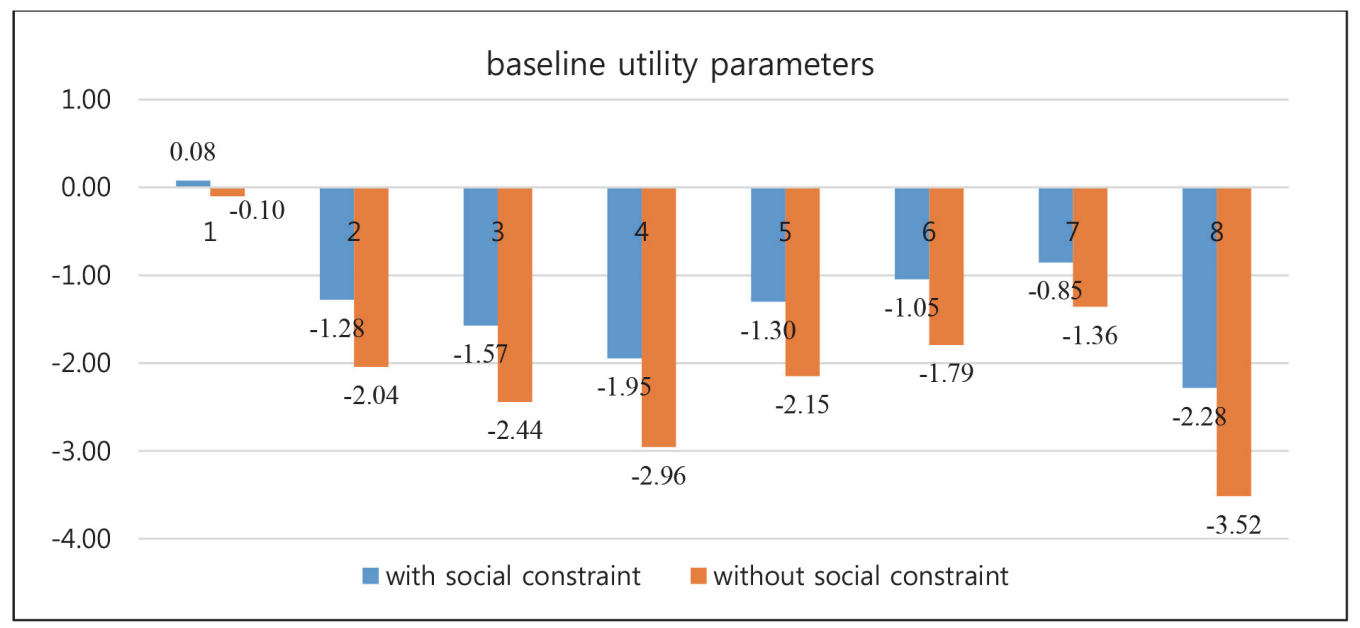

which would eventually lower the preference parameter estimates. Figure 2 shows the amount and the pattern of bias. The first shirts $\left(\mathrm{x}_{1}\right)$ having attribute combination $\left(a_{1}=0, a_{2}=0\right)$ close to social norm shows the least difference in baseline preference estimates.

This bias is also observed in the attribute impact parameters. The estimates of $\left(\overline{\beta_{1}}, \overline{\beta_{2}}\right)$ is $(-0.873-1.066)$ for proposed model and $(-1.418-1.645)$ for benchmarks. Although consumers do not prefer anyway having colored and patterned shirt relative to white and non-patterned shirt, benchmark model overly states this low preference. It is because any lower demand in the data is to be attributed to consumers' preference only in benchmark model whereas the proposed multiple constraint model separates the high pressure from low preference, resulting in the preference parameter estimates not as low as single constraint model depicts.

\section{Managerial Implication}

The estimation results show that the observed demand is more consistent with the multiple constraint model, indicating the meaningful role of social norm in consumer decisions on shirt choice. Three components in the model that contributes to demand for a product are social burden, monetary constraint, and consumer's preference. Thus, marketers need to know whether or not it's worthwhile to pay attention to the information about social burden more than to preference estimates or to price constraint. From now on, we discuss managerial implication of the proposed model from marketer's view point. 
5.1 Social norm versus preference as a sales predictor

In figure 3 , the coordinates for each of the eight shirts used in the data are given based on the mean preference and social burden, i.e., $\left(\overline{\psi_{n}}, w_{n}\right)$ for $n=1, \ldots, 8$. The plot reveals a pattern that the amount of social burden and the estimated preference are negatively correlated. Products with high(low) preference appear low(high) in social burden. Thus, marketers need to know which one is more compelling reason to make people buy(not buy) the product - high(low) preference or low (high) social burden.

To address this question, we calculate the values of the relative sales among products and check whether these numbers are closer to or consistent with the preference $\operatorname{ratio}(\psi)$ and social burden ratio $(w)$. Without loss of generality, we fix the first shirt as a reference good and calculate the following quantities relative to this reference for the rest of shirts $(\mathrm{n}=2, \ldots, 8)$ :

Sales ratio $_{n}=\frac{\text { sales }_{n}}{\text { sales }_{1}}$

Preference ratio $_{n}=\frac{\psi_{n}}{\psi_{1}}$

Social burden ratio $_{n}=\left(\frac{w_{n}}{w_{1}}\right)^{-1}$

The pattern of these indices are given in figure 4. In order to make the directions comparable among indices, social burden ratio is given in inverse form. From the plot, it is obvious that relative ratio of social burden between products $\left(\frac{w_{1}}{w_{n}}\right)$ is much more informative

〈Figure 3〉Social burden $(w)$ and baseline preference $(\psi)$ for products

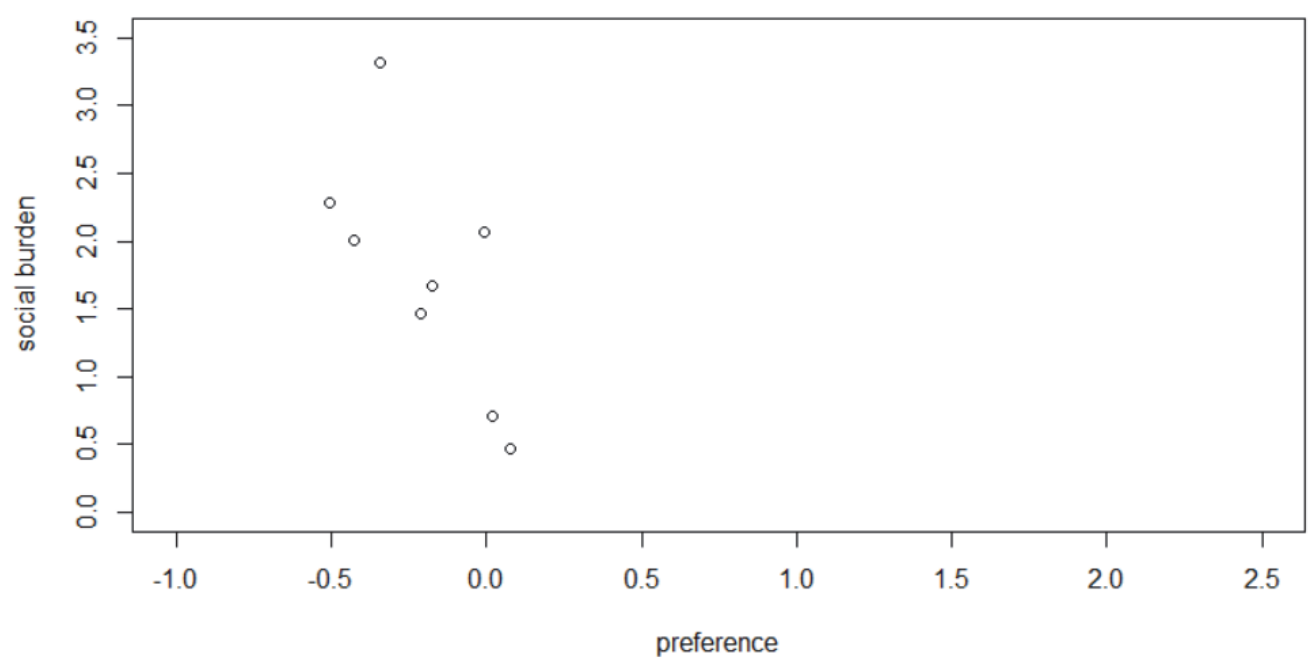


〈Figure 4〉 Sales ratio, preference ratio and social burden ratio (reference good: alternative 1)

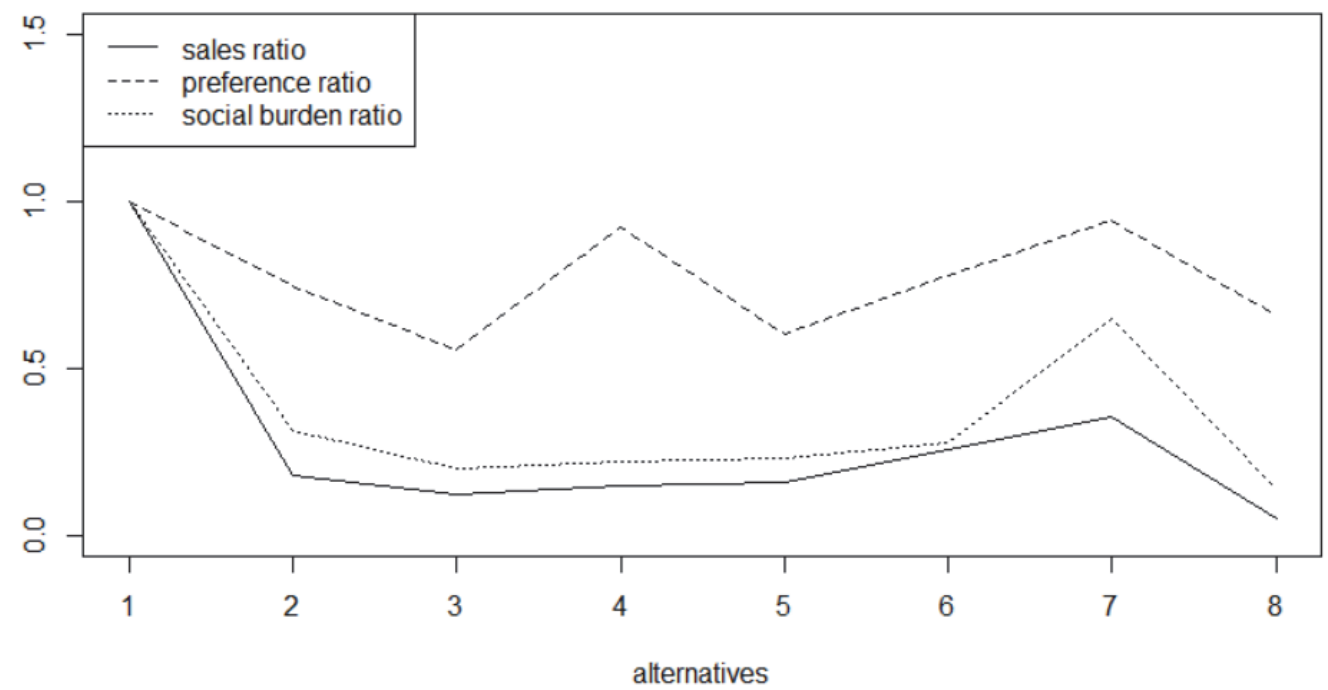

about product sales than is the preference ratio $\left(\frac{\psi_{n}}{\psi_{1}}\right)$.

\subsection{Consumer welfare impact of social norm versus price discount}

In this part, we compare the policy impact on maximum attainable utility between social cost and monetary cost. Product presented with either price reduction or lowered social burden is an attractive option to a consumer, and it would increase the indirect utility. Then, which policy has stronger impact on the welfare change? We quantify the effect by evaluating the changes in indirect utility as marketers offer price cut or reduction in social cost.

For counterfactuals, we first evaluate the indirect utility using the posterior draws of the model parameters.

$$
\bar{V}^{(0)}=\sum_{k=1}^{K} \sum_{t=1}^{T} V_{k t}\left(p_{k t}, w_{k n}\right)
$$

Then, we explore the changes in this aggregated welfare as we relieve one constraint by stepwise discount while holding the other constraint unchanged. For simple evaluation, we use five grids $-5 \%, 10 \%, 20 \%, 30 \%$, and $40 \%$ for each computation.

$$
\begin{gathered}
\bar{V}\left(r^{(w)}\right)=\sum_{k} \sum_{t} V_{k t}\left(p_{k t},\left(1-r^{(w)}\right) \cdot w_{k}\right) \\
\text { for } r^{(w)}=.05, .10, .20, .30, .40 \\
\bar{V}\left(r^{(p)}\right)=\sum_{k} \sum_{t} V_{k t}\left(\left(1-r^{(p)}\right) \cdot p_{k t}, w_{k}\right) \\
\quad \text { for } r^{(p)}=.05, .10, .20, .30, .40
\end{gathered}
$$

where $r^{(w)}$ and $r^{(p)}$ are discount depth for 
social burden $(\boldsymbol{w})$ and price $(\boldsymbol{p})$, respectively.

The impact from each of the policy change was computed as $\frac{\bar{V}^{(0)}-\bar{V}\left(r^{(w)}\right)}{\bar{V}^{(0)}}$.

Table 6 shows how much the consumer welfare changes for each policy change and the relative strength of the impact between the two policies. We find that the consumers' benefit from relieving social norm is higher than the impact from price cut. For instance, $10 \%$ reduction from the current social cost $\left(r^{(w)}=.10\right)$ increases the maximum attainable utility by $4 \%$ while the same percent of price reduction $\left(\left(r^{(p)}=.10\right)\right)$ increases the welfare by $1.22 \%$. This differential impact is held across all five policies. As reported in the last column in table 6, on average, the welfare effect of norm is three times bigger than price cut.

Figure 5 shows these results more intuitively. As discount increases, the difference between the two policies becomes larger. From marketers' perspective, any marketing programs from

〈Table 6〉 Welfare impact: reduction in price vs social pressure

\begin{tabular}{rccc}
\hline & $\begin{array}{c}\text { Price discount } \\
(\mathrm{A})\end{array}$ & $\begin{array}{c}\text { Reduction in } \\
\text { psychological burden(B) }\end{array}$ & $\begin{array}{c}\text { Ratio } \\
(\mathrm{B} / \mathrm{A})\end{array}$ \\
\hline $5 \%$ reduction & $0.58 \%$ & $1.83 \%$ & 3.13 \\
$10 \%$ reduction & $1.22 \%$ & $4.00 \%$ & 3.23 \\
$20 \%$ reduction & $2.48 \%$ & $8.63 \%$ & 3.45 \\
$30 \%$ reduction & $4.03 \%$ & $14.08 \%$ & 3.45 \\
$40 \%$ reduction & $5.76 \%$ & $20.81 \%$ & 3.57 \\
\hline
\end{tabular}

〈Figure 5〉 Welfare change from price discount and reduction in social burden

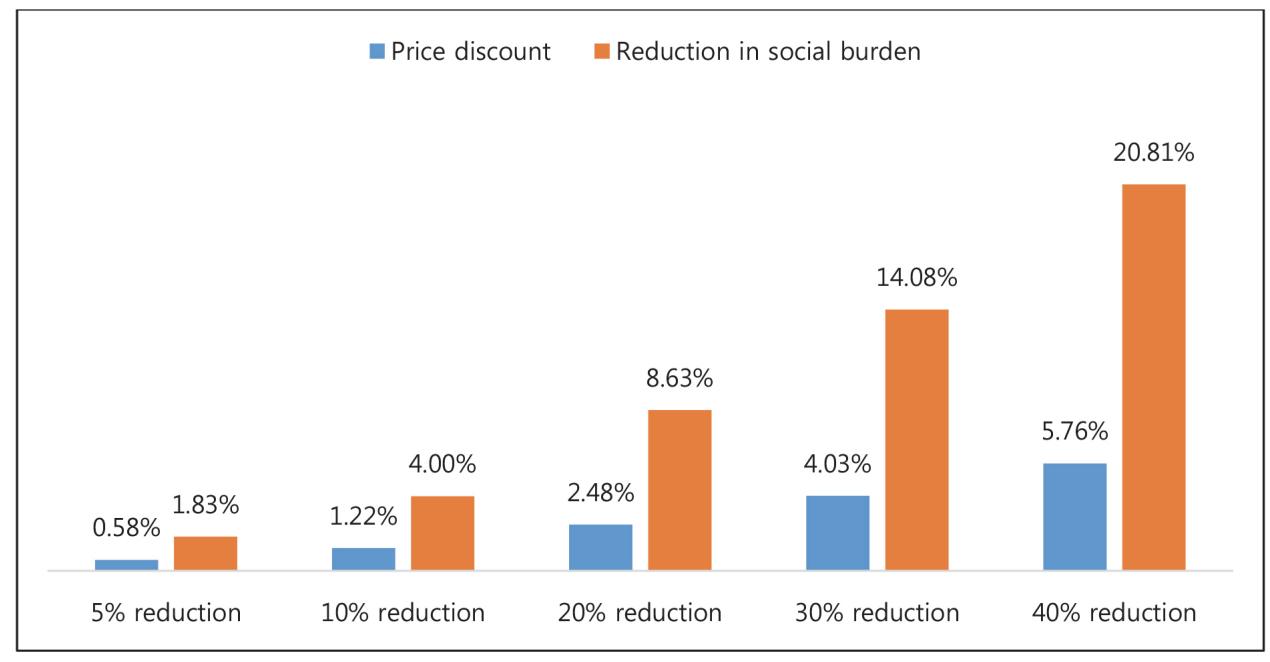


product configuration to promotional communication that offers even small changes in consumer conformity to social norm can be as good as a large price discount. For example, a 30\% price discount shows the similar result with $10 \%$ social burden reduction, i.e., about $4.0 \%$ welfare impact.

\section{Concluding Remarks}

A consistent body of research in social science area has shown that human behavior is affected by the social environment. Individuals make their own decisions, yet their behavior often reflect the influences from these external force in a wide range including eating, wearing, learning, talking, and so on. Buying and consuming in market places are not exempt from this influence as a consumer is also one of the entities to go through social interaction. Social norms set an expectation on consumer purchase decision.

The goal of this study is to model the role of social norm in consumer demand where purchase decisions are not buying single-item-only, but can be multiple-item-buying as well. Based on economic utility framework, we allow for social norm to influence demand as additional constraint in consumers' utility maximizing decision. The presence of additional constraint in consumer decision making restricts the feasible region for non-zero demand. Therefore, low demand or no-purchase for any good can come from not only lower preference but also from higher cost associated with constraint consumers have. The model was applied to conjoint data from office workers' choice of shirts at work place.

Key findings from the empirical analysis are as follow. First, the proposed model with social constraint fits better than the benchmark model with budget constraint, which supports that the effect of social norm is not trivial even after accounting for preference effect. Second, the estimates for preference parameters suffer from downward bias when ignoring social norm in model specification. Third, both preference and norm-related parameters turn out to be substantially heterogeneous across individuals, but such variation was not related to individuals' age and gender. Fourth, work experience variable - one of the individual covariates, is positively related to individuals' psychological capacity parameter that enables consumers to tolerate pressures from social norm.

The study also offers contributions to marketers. First, practitioners in the field need to have good outlook for the variation in their marketing performances. Unlike common belief that consumer preference or its relative strength to competitors determines the sales performance in the market, the sales of the shirts were a lot more consistent with the level of conformity to social norms associated with each shirt. Secondly, in an effort to search for tools and programs to 
shift consumer welfare, this study provides an important intuition to policy decision makers. It is that working on programs that mitigates the consumer's concern from social norm is in fact very effective and the calculated effect on consumer welfare is about three times bigger than promotional price cut.

There are several interesting avenues for future research. First, some consumers may have desire for uniqueness more than conformity, although most people perceive discomfort when they are away from social norm. We think that the uniqueness versus conformity is not the constructs lying on the same dimension with different position, but they are more of multi-dimensional. In this study, we allow for conformity only, so this can be extended. Incorporating uniqueness into constraint is challenging, yet would offer important learning on norm effect. Second, there are probably other individual characteristics that help customers stand robust to the expectation from the norm. In our analysis, only 'work experience' was significant. Possible variables are type of social interaction that individuals usually have had, occupation (in that work experience is characterized in this domain), family size(larger family might offer better opportunity to expose one to others' thoughts), etc. These variables can be readily incorporated though the hierarchical model of the current study. The application of the current model to other categories and contexts may alter the empirical estimates including the positive role of job experience in making consumers relatively robust to social norm. Asia is still a growing market, and this market is known to be more sensitive toward the social norms, other people's gaze and evaluation. Identifying variables responsible for high and low sensitivity to norm will certainly help global companies looking for efficient targeting and market segmentation-base variables tailored to Asian consumers. We leave these topics for future research.

〈Received February 2. 2020〉

〈Accepted March 31. 2020〉

\section{References}

Ajzen, I., \& Fishbein, M. (1973). Attitudinal and normative variables as predictors of specific behavior. Journal of Personality and Social Psychology, 27(1), 41-57.

Allenby, G. M., P. E. Rossi, R. E. McCulloch (2005), "Hierarchical bayes models: a practitioner's guide," SSRN Working Paper.

Baron, S. Robert, Norbert L. Kerr, and Norman Miler (1992), "Group process, group decision, group action," Open University Press.

Bhat, C.R. (2005), “A multiple discrete-continuous extreme value model: Formulation and application to discretionary time-use decisions," Transportation Research, 39(8), 679-707. 
Bhat, C.R. (2008), "The multiple discretecontinuous extreme value (mdcev) model: Role of utility function parameters, identification considerations, and model extensions," Transportation Research, 42 (3), 274-303.

Brewer, B. Marilynn, and Miles Hewstone (2004), "Social Cognition (Perspectives on Social Psychology)," Blackwell.

Fisher, J. Robert, Ackerman David (1998), "The Effects of Recognition and Group Need on Volunteerism: A Social Norm Perspective," Journal of Consumer Research, 25(3), 262-275.

Gockeritz, Susanne, P. Wesley Schultz, Tania Rendon, Robert B. Cialdini, Noah J. Goldstein, and Vladas Griskevicius (2010), "Descriptive normative beliefs and conservation behavior: the moderating roles of personal involvement and injunctive normative beliefs," European Journal of Social Psychology, 40(3), 514-523.

Haines, M, SF. Spear (1996), "Changing the perception of the norm: a strategy to decrease binge drinking among college students," Journal of American College Health, 45(3), 134-140.

Harries, Tim, Ruth Rettie, Matthew Studley, Kevin Burchell, Simon Chambers (2013), "Is social norms marketing effective?: A case study in domestic electricity consumption," European Journal of Marketing, 47(9), 1458-1475.
Kim, Byungyeon, Takuya Satomura, and Jaehwan Kim (2017), "A direct utility model with dynamic constraint," Asia Marketing Journal, 18(4), 125-138.

Kim, Jaehwan, Greg M. Allenby, and Peter E. Rossi (2002), "Modeling consumer demand for variety," Marketing Science, 21(3), 229-250.

Kim, Jaehwan, Greg M. Allenby, and Peter E. Rossi (2007), "Product attributes and models of multiple discreteness," Journal of Econometrics, 138(1), 208-230.

Kreps, M. David (1997), "Intrinsic motivation and extrinsic incentives," The American Economic Review, 87(2), 359-364.

Lee, Sanghak, Jaehwan Kim, and Greg M. Allenby (2013), “A direct utility model for asymmetric complements," Marketing Science, 32(3), 454-470.

Leon, Festinger, Schachter Stanley, and Back Kurt (1950), "Social pressures in informal groups; a study of human factors in housing," Oxford, England: Harper.

Lin, Chen, Sriram Venkataraman, and Sandy D. Jap (2013), "Media multiplexing behavior: Implications for targeting and media planning," Marketing Science, 32(2), 310324.

Luo, Lan, Brian T. Ratchford, and Botao Yang (2013), "Why we do what we do: A model of activity consumption," Journal of Marketing Research, 50(1), 24-43.

Paluck, Elizabeth Levy, and Laurie Ball (2010). 
"Social norms marketing aimed at gender based violence: A literature review and critical assessment," New York: International Rescue Committee.

Richa, Yadav, Wermter Stefan (2009), “Book review: social cognition, Marilynn B. Brewer, Miles J. Hewstone (Eds.); Blackwell Publishing, 2004, 368 pages," Cognitive Systems Research, 2009 10(2), 172-174.

Rossi, P. E., G. M. Allenby, and R. McCulloch (2005), "Bayesian Statistics and Marketing," John Wiley \& Sons, Ltd.

Satomura, Takuya, Jaehwan Kim, and Greg M. Allenby (2011), "Multiple-constraint choice models with corner and interior solutions," Marketing Science, 30(3), 481490.

Sen, Sankar, Zeynep Gürhan-Canli, Vicki Morwitz (2001), "Withholding Consumption: A Social Dilemma Perspective on Consumer Boycotts," Journal of Consumer Research, 28(3), 399-417.

Sheikh, Afzal, Sunil Vadera, Ichael Ravey, Gary Lovatt, Grace Kelly (2017), “A social norms approach to changing school children's perceptions of tobacco usage," Health Education, 117(6), 530-539. 